ÁMBITOS. № 17 - Año 2008 (pp 25-36)

\title{
LOS EFECTOS DE LAS REDES CIUDADANAS EN LA CAMPAÑA ELECTORAL DEL 9-M
}

\author{
Guillermina Franco Álvarez \\ gfranco@hum.uc3m.es \\ David García Martul \\ dgmartul@bib.uc3m.es \\ (Universidad Carlos III)
}

\section{Resumen:}

La campaña electoral del 9-M en España ha propiciado desde los medios digitales y audiovisuales un espacio de convergencia tecnológica orientada a potenciar la difusión de los mensajes electoralistas y a crear un espacio nuevo de promoción desde las nuevas plataformas emergentes, las redes ciudadanas. He aquí que la materia de nuestra investigación se centre en los efectos y herramientas que son utilizados por los partidos políticos con mayor representación parlamentaria. Para ello, repasamos alguna de las tecnologías empleadas durante esta campaña y su repercusión en el espacio digital. Así pues, proporcionaremos una metodología centrada en la observación del uso que se ha hecho de estas tecnologías durante la última campaña electoral. La metodología empleada ha sido el análisis cualitativo extraído de las entrevistas y los informes obtenidos de los distintos gabinetes de comunicación y los representantes implicados en la campaña institucional del PP (Partido Popular) y el PSOE (Partido Socialista Obrero Español).

Palabras clave: Multimedia, tecnología digital, redes ciudadanas, espacio político, nuevos medios.
Abstract:

The election campaign of 9-M in Spain has led since digital media and audiovisual an area of convergence of technologies aimed at enhancing the dissemination of messages electioneering and create a new space advocacy electioneering from new platforms emerging civic networks. Behold the subject of our investigation focuses on the effects and tools that are used by political parties with greater parliamentary representation. To that end, we look at some of the technologies employed during this campaign and its impact on the digital space. Thus, we will provide a methodology focused on observing the use made of these technologies during the last election campaign. The methodology has been qualitative analysis drawn from interviews and reports from the various cabinets communication and representatives involved in the campaign's institutional PP (Partido Popular) and the PSOE (Spanish Socialist Workers' Party).

Keywords: Multimedia, digital technology, citizen networks, policy space, new media. 


\section{INTRODUCCIÓN}

L

a lucha bipartidista PSOE y PP en esta última campaña electoral ha promovido, por parte de ambos partidos políticos, iniciativas de plataformas interactivas digitales y audiovisuales con la finalidad de captar votantes de otros perfiles de edad adaptados a entornos digitales, entre los que destacan los jóvenes. Tal y cómo señalan Dubin y Kalsow (1996) se piensa que si el modo de votar resulta más cómodo y flexible, aumentará el número de votantes. La hipótesis primordial es que desde las cavernas de los propios partidos la captación de un público interesante y poco ávido de las actividades políticas como son los jóvenes, supone un perfil seductor de población con el que contar para la captación del electoral. Mientras, en otros países como EE.UU. se han creado alternativas para aumentar el voto por Internet, como es el caso del grupo de trabajo de California dedicado al voto por Internet (California Internet Voting Task Force), compuesto por funcionarios del gobierno, expertos en informática y en seguridad y en organizaciones de votantes sin filiación política, que se creó para determinar la viabilidad del voto por Internet. Asimismo, este estudio define una estrategia de seguimiento y acceso a aquellos ciudadanos que no teniendo una afiliación ideológica y política se concentran en escuchar las voces y mensajes virtuales de los líderes políticos a través de nuevas herramientas interactivas, más próximas y más analíticas para el ciudadano. Este público, representado en su mayoría por jóvenes votantes sin filiación política e ideológica supone un segmento poblacional importante de seducción para las distintas alternativas políticas.

Por lo tanto, en esta investigación lo que se plantea es el efecto de estas redes sociales en la población y el uso de estas plataformas multimedia. Las plataformas virtuales en muchos casos alternativas han provocado que los partidos políticos consoliden su modus operandi de promoción y representación ideológica. Para ello, presentaremos los diferentes indicadores que han participado en este contexto político multimediático virtual.

\section{NUEVOS MEDIOS Y CONVERGENCIA}

A menudo surge el debate o la discusión académica de por qué los nuevos medios se denominan así y por qué alguno se ha considerado esta denominación de nuevo. Estos medios son considerados así porque existe una lista de tecnologías llamadas nuevas pero que se encuentran en continuo cambio. Es el caso de la tecnología de vídeo digital (DVD), si la comparamos con el grabador de video casette (VCR), aunque de aparición menos novedosa si la comparamos con el compact disk (CD). Una aproximación de nuevos medios y que cataloga las tecnologías por sí mismas, cuestionando el contexto social y cultural. En esta dinámica la teórica Sonia Livingstone apunta: ¿Qué es nuevo para la sociedad en relación a lo que denominamos nuevos medios? Otra cuestión que se formula es, ¿Qué son los nuevos medios? (Livingstone, 1999). Definimos pues como nuevos medios aquellas formas que combinan las tres C's de lo que entendemos por medios convergentes. 
Realmente si tuviéramos que encontrar una definición de lo que significa nuevo medio entendido como aquel que combina las tres C's: tecnologías de la información y la computación (IT), las redes de comunicación, y los contenidos de medios digitales e informativos (Miles, 1997), en mitad de los tres círculos nos encontramos el elemento común de convergencia, Internet y la World Wide Web. En esta línea de pensamiento nos adscribimos a las teorías de Lievrouw y Livingstone (2002) en sus observaciones de las aproximaciones planteadas acerca de lo que necesitan los nuevos medios para tomar en cuenta estos tres elementos:

1. Los artefactos o dispositivos de los que disponemos prolongan nuestra capacidad para comunicar.

2. Las actividades y prácticas comunicativas nos atraen en el desarrollo y uso de estos dispositivos.

3. Instituciones y organizaciones suponen un comportamiento alrededor de estas prácticas y dispositivos.

Asimismo, el enunciado de estos nuevos medios se plantea el concepto de digital media. Los medios digitales abarcan formas de contenidos en medios de comunicación que combinan e incorporan textos, datos, sonido e imágenes en gran número de formatos. El uso y la extensión adaptada de estos formatos y la distribución de esta información en redes de información contribuyen a que las redes ciudadanas sean cada vez más extensas. Otro aspecto es que cada medio tiene la característica de ser manipulable, la información digital es fácilmente canjeable y adaptable, todos la almacenamos, la distribuimos y la usamos.

Otro indicador a tener en cuenta es la readaptabilidad-la información digital puede ser compartida e intercambiada entre el mismo número de usuarios simultáneamente, atravesando enormes distancias. La comprensibilidad también es otro factor inherente. La mayor capacidad que la información digital tiene en la red permite que pueda ser tanto comprimida como descomprimida cuando el usuario así lo necesite.

De todas las características enumeradas nos encontramos con una última, la imparcialidad -la representación de la información digital es indiferente a través de la red, circula en función de quiénes la utilicen y es usada por otros usuarios. Por ejemplo, un pasaje de la Biblia, un artículo académico, o una imagen pornográfica y las noticias de un vídeo separan el espacio y la imagen de la CNN en red, o los servicios de noticias internacionales de la BBC, todos son simplemente una combinación de ceros y unos en un entorno digital (Feldman, 1997).

En este sentido la idea del nuevo medio desarrolla una única forma de comprender los medios digitales y recuerda cómo los medios tradicionales han sido adaptados $-\mathrm{y}$ adoptados- a las nuevas tecnologías. Es frecuente que los contenidos de estos medios hayan cambiado a lo largo de estos años, lo que comenzó con el llamado Periodismo 1.0 (se define como aquél que traspasa contenidos tradicionales a la Red), ha ido desarrollándose y buscando otra fórmula que alcanza un nivel 2, llamado Periodismo 2.0. En éste intervienen elementos partícipes de la interactividad: fotografías, hipertexto, hipermedia, etc., en definitiva todos aquellos elementos que 
se unen en una única plataforma llamada multimedia. Esta plataforma multimedia y el uso de la Web Social 2.0 tienen como cliente o usuario más próximo a los jóvenes, según la EGM ${ }^{1}$. Ello ha permitido a los partidos políticos durante la campaña electoral del 9-M, la creación de plataformas interactivas que les animen a interactuar, relacionarse, opinar y además garantizar el ciudadano participativo activo, un recurso que le proporciona al político obtener y capturar simpatizantes durante la campaña electoral.

Pero el ciudadano no reclama únicamente su participación. Los medios digitales en España han ido acelerando este proceso de participación a través de la incorporación de nuevas herramientas interactivas que ayudan al ciudadano a formar parte de ese espacio virtual que se define como la sociedad invisible:

Los medios de comunicación suscitan una familiaridad y proximidad con las cosas y las personas, pero no permiten ver la otra cara de la realidad: su manufactura, su carácter de mediación construida, su superficialidad. La visibilidad y transparencia de los medios producen una ceguera específica: la profusión de las imágenes y palabras saturan con una masa indiferenciada de hechos brutos (Innerarity, 2004).

La originalidad de las nuevas formas del secreto está en su hipervisibilidad. Como en la carta robada de Poe, para construir un secreto no es necesario ocultar, basta con publicitar y mostrar (Moraza, 2002).

\section{INTERACTIVIDAD Y MECANISMOS DE PARTICIPACIÓN}

La interactividad es un concepto central entendido y adoptado por los nuevos medios, a pesar de que los formatos existentes en los medios poseen diferentes grados de interactividad, a pesar de que muchos de los formatos existentes en los medios actuales no suponen una realidad interactiva del todo. En este sentido Sheiza Rafaeli define interactividad como la medida en que la comunicación refleja un nuevo eje en sí misma, se alimenta y responde a estos últimos (Newhagen y Rafaeli, 1996). A este respecto, Internet establece una comunicación con un grado muy alto de interactividad, desde el acceso y contenidos online, creación de archivos, bases de datos y nuevas formas de distribución y elaboración de contenido, como es la creación de un archivo que contribuya a crear listas de discusión. Pero realmente la interactividad más reconocida en Internet es conocida como la comunicación persona a persona, representativo de ello es la aplicación conocida por chat.

Internet implica la comunicación persona a persona, comunicación en grupo, y publicidad y en algunos casos también propaganda. Esta propaganda virtual ha sido lanzada a la Red por los partidos políticos, esta cualidad mística de la que habla Gibson (1994) hace especial referencia al ciberespacio como una alucinación consensual en Neuromancer. Durante esta campaña en la red se han fomentado los elementos interactivos que ayudan a crear un nuevo modelo de intercambio de información, de las que forman parte disciplinas como el HCl (Human Computer Inter-

\footnotetext{
1 Según la última ola de octubre/noviembre de 2007 de la Encuesta General de Medios (EGM), el perfil de edad con tasa más alta de penetración de Internet se encuentra en el intervalo de 25 a 34 años.
} 
action). Esta relación de lo que es interactividad debatida en un amplio número de foros realza que los elementos a disposición del usuario (los recursos interactivos) son controlados de forma no directa por el medio que los incorpora. El estímulo virtual $^{3}$ abierto a la participación ciudadana ha sido recogido y lanzado por los partidos políticos con una intención, la de captar más votantes afines y abrir la participación a otra tipología de perfil. Los recursos interactivos empleados, las plataformas de redes ciudadanas y sus efectos es lo que en definitiva centra esta investigación.

Esta mal llamada publicidad, más cercana a la propaganda electoral, le ha servido a los partidos políticos para utilizar el medio digital como un fiel exponente proclive a la tecnología y para convertir el medio en un instrumento indiscriminado utilizado por la masa:

La tecnología digital ha permitido romper el patrón del broadcasting, la comunicación de masas, de uno a muchos, y sustituirla por un modelo distribuido en el que muchas personas pueden dirigirse unas a otras estableciendo un diálogo en enriquecimiento donde se van juntando piezas para componer un mensaje complejo (Varela, 2005).

\subsection{Los mecanismos de participación}

La captura del voto electoral ha obligado a los partidos políticos a incorporarse rápidamente a las tecnologías digitales. La inmediatez ${ }^{4}$ de Internet es un factor decisivo para poder efectuar el seguimiento de la campaña en todo momento con total ubicuidad. Esta fórmula se encuentra representada en medios y plataformas de comunicación digitales. Para ello, algunos portales han llevado a cabo un esfuerzo adicional con el fin de potenciar estas características. Se ofrecen herramientas tan diversas como originales, con las que se facilita la actualización de los contenidos por parte de sus usuarios y potenciales votantes. Un ejemplo es este portal http://noticias.msn.es/elecciones/elecciones $2008 /$ messenger.

\footnotetext{
2 Alucinación consensual es el término definido por William Gibson en Neuromancer o Neuromante (en español) (1984), una novela de ciencia ficción por este mismo autor. Primera obra, y es una de las pocas obras en haber obtenido los tres premios más importantes en la literatura de ciencia ficción: el Premio Nébula, el Premio Hugo y el Premio Philip K. Dick. Neuromante es la novela principal de la corriente denominada cyberpunk.

3 Llámese a los recursos interactivos disponibles en los medios y portales informativos para fomentar la interactividad y la participación digital durante la campaña electoral del 9-M.

4 Término muy extendido entre los periodistas digitales y los medios en línea.
} 
Imagen 1. Portal de buddies durante las elecciones generales del 9-M de Messenger.

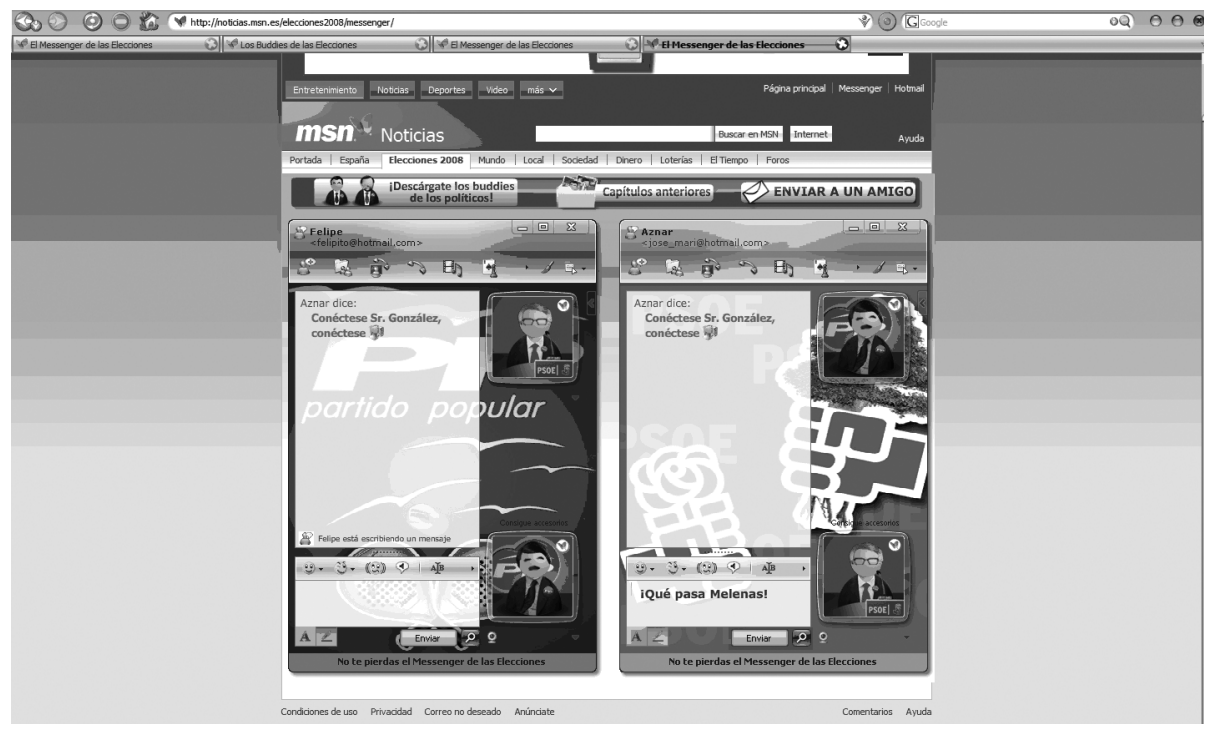

Fuente: http://noticias.msn.es/elecciones2008/messenger/

Estas páginas proporcionan conversaciones en línea entre los dos candidatos sobre temas de debate como es el tren de alta velocidad o los problemas con el AVE Madrid-Barcelona. Se trata de una de las numerosas iniciativas que ha puesto en marcha el portal de noticias online MSN con motivo de las elecciones, con contenidos originales y novedosos entre los que se encuentran el uso de buddies ${ }^{5}$, colegas usados en la plataforma interactiva del chat.

Si bien se aplican preferentemente a conversaciones a través de mensajes escritos, también existe otra tipología de chat que incorpora sistemas de código audiovisual, en conversación en línea, audio y vídeo. El uso de esta herramienta interactiva se extiende tanto a una herramienta de trabajo como de ocio, el uso por los ciudadanos es cada vez más asiduo, y su portal representativo popularizado por Microsoft es el que figura en esta dirección electrónica http://messenger.msn.es/download. Este portal ha contribuido en la campaña electoral española a crear una alternativa novedosa el seguimiento de las discusiones de manera ficticia en Messenger entre el presidente del gobierno, José Luis Rodríguez Zapatero y el líder de la oposición, Mariano Rajoy sobre los temas centrales de la campaña.

A este espacio de virtualidad abierta en campaña se unen otros portales interactivos como www.discursia.com, que presenta una relación de diputados por partido, destacando entre sus enlaces las palabras o frases más pronunciadas por los políticos.

\footnotetext{
5 También denominado colega. Los buddie's son los personajes del Messenger disfrazados con diferentes temáticas. En la página encontrarás varios buddie's y puedes escoger el que más te guste. Luego, tienes que descargarte un .zip donde viene la imagen de 96×96, que debes guardar en tu máquina. Disponible en http://amigomensajero.com [Consulta: 1 de febrero de 2008].
} 
Imagen 2. Información relativa a los diputados de la IX Legislatura con información sobre su actuación política.

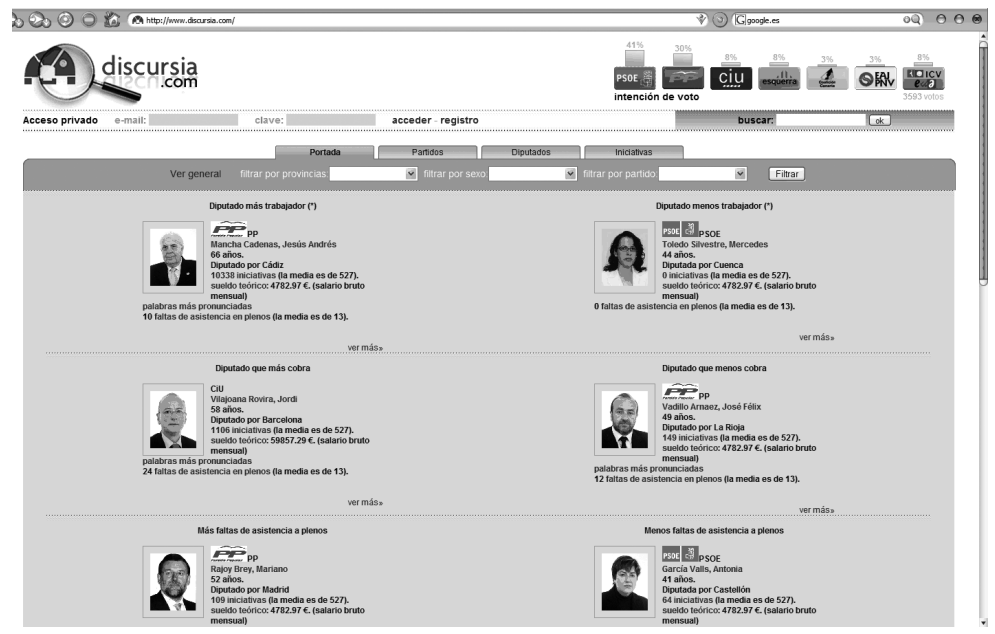

Fuente: www.discursia.com

Paralelamente a estas alternativas comparecen otras. Un ejemplo claro es la alternativa del portal www.portalelectoral.es. Este sitio ofrece un listado de opiniones y enlaces derivados de diferentes personajes del mundo de la política, el arte y la cultura entre otros.

Pero junto a esta atmósfera virtual nos encontramos con la Web 2.0 que cuenta con numerosas definiciones. Tim O'Reilly plantea la Web 2.0 como un negocio y plataforma global que convierte la Web en un sitio abierto. Este entorno ha planteado la construcción de aplicaciones y servicios en torno a las características singulares de Internet. La frase Web 2.0 alude a una forma mejorada de la World Wide Web. Tecnologías, como los weblogs (blogs), wikis, podcasts, feeds RSS (y otras formas de muchos-a-muchos de publicación), software social, y la Web de interfaces de programación de aplicaciones (API) proporcionan mejoras más de los sitios web de sólo lectura.

Por otro lado, la Interactividad se entiende como 2.0

todas aquellas utilidades y servicios de Internet que se sustentan en una base de datos, la cual puede ser modificada por los usuarios del servicio, ya sea en su contenido (añadiendo, cambiando o borrando información o asociando datos a la información existente), bien en la forma de presentarlos, o en contenido y forma simultáneamente (Ribes, 2007).

En este sentido la Web 2.0 no ha quedado al margen de la carrera electoral. Según datos del Gobierno, alrededor de 20 millones de usuarios acceden en España a Internet $y$, de ellos, el $41 \%$ lo hacen a través de redes de banda ancha. Este resultado ha favorecido la participación e interactividad con los usuarios: 
Los sinónimos usados en conexión con la llegada de los nuevos medios, han llegado a ser términos extendidos en el campo de estudios de medios de comunicación. En este sentido, el concepto de interactividad o su combinación de medios interactivos es más a menudo utilizado para caracterizar un cierto rasgo de nuevos medios el cual establece la diferencia con los medios tradicionales. (Franco, 2005).

Quizás de entre todos los recursos de valor añadido empleados en la campaña electoral digital podríamos destacar el código audiovisual interactivo ${ }^{6}$ o vídeo interactivo como el más favorecido en la misma. Este recurso, propiedad desde 2006 del motor de búsqueda más extendido en Internet, Google, usa un reproductor en línea de Adobe Flash. Su facilidad y popularidad se sostiene por la posibilidad de alojar vídeos personales de forma sencilla. El índice de visitas y su aceptación por parte de la población ha sido tan relevante que las cadenas televisivas se han sumado a ello, un ejemplo es el caso de RTVE.es

Figura 3. La difusión de información electoral de RTVE en YouTube.
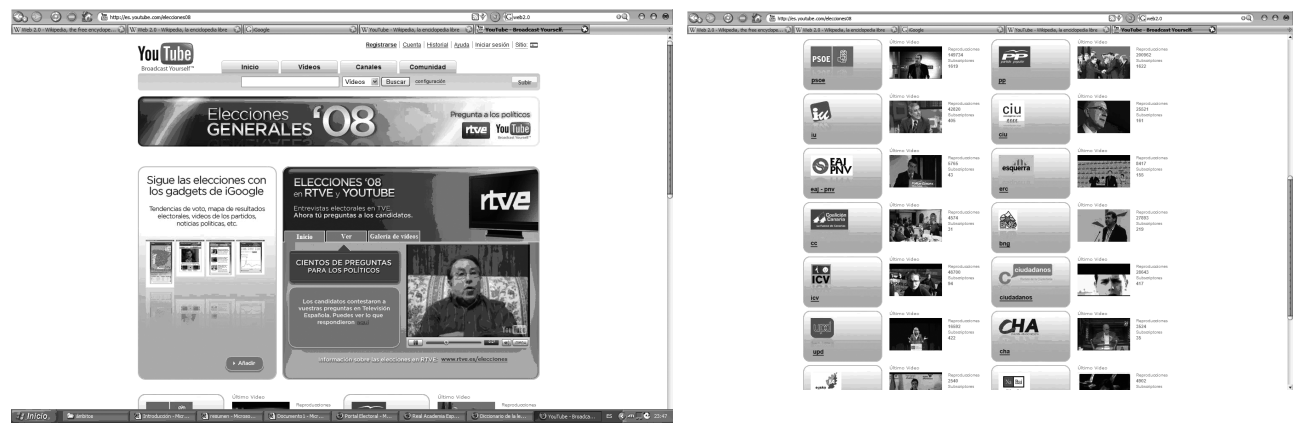

Fuente: www.youtube.es/elecciones08

Pero no sólo esta cadena se ha visto asediada por la participación electoral, donde más de seiscientos ciudadanos han participado colgando sus vídeos con su pregunta al político. Otra iniciativa alternativa la planteaba la cadena Antena $3^{7}$. La cadena televisiva Sexta ${ }^{8}$, también se sumó con las posibilidades que ofrecía YouTube, con la finalidad de que los usuarios pudieran realizar y publicar sus preguntas a los líderes de cada partido. Estas iniciativas han favorecido la disponibilidad de una plataforma y el acceso por parte del ciudadano de comentarios, ideas y proyectos de los dirigentes de las distintas alternativas políticas. YouTube ha supuesto un recurso de estreno para esta campaña electoral donde la participación ha sido muy alta, tanto en la elaboración de vídeos con planteamientos personales como la participación en la red.

\footnotetext{
${ }^{6}$ Se define por los autores de este artículo como código audiovisual interactivo aquel servicio de valor añadido de soporte audiovisual.

7 Disponible en http://es.youtube.com/elecciones9m [Consulta: 6 de marzo de 2008].

8 Disponible en http://www.tuspreguntas.com [Consulta: 6 de marzo de 2008].
} 
Junto a este servicio de la Web 2.0, el gran protagonista de esta etapa lo ha caracterizado el mundo de los Weblogs (blogs o bitácoras), a los que Varela (2005) define como agitadores y promotores de la movilización, [que] hicieron su entrada oficial en la vida pública. Aparecieron ante los medios, la política y los ciudadanos como lo habían hecho antes entre las ruinas de las Torres Gemelas, el 11-S de 2001, y la invasión de Irak, cuando los warlogs ${ }^{9}$ se convirtieron en la fuente informativa alternativa. Estas agendas temáticas personales han irrumpido en la vida de los ciudadanos y también han ofrecido durante esta campaña una diversidad informativa.

Antes de los blogs apareció Freenet. Esta red se destacaba como una red de comunicaciones entre pares, más conocidas como P2P (Peer-to-peer), descentralizada y diseñada para resistir la censura, que utiliza el acho de banda y espacio de almacenamiento de las computadoras de sus miembros para permitir publicar u obtener información de todo tipo en completo anonimato ${ }^{10}$.

En este sentido, los teóricos argumentan:

Era evidente que convertirse en un gran medio electrónico, como son las versiones online de Clarín, La Nación o El País estaba totalmente fuera del alcance del usuario común. Por eso, sólo la invención de los Weblogs pudo remover las barreras técnicas de la publicación para un gran número de personas. Desde que existen los blogs somos -con mayor o menor fortuna- nuestros propios contenidistas, editores, asistentes técnicos, diseñadores, etc. (Piscitelli, 2005).

Esta gran revolución de los cuadernos o libretas personales electrónicas ha tenido su reflejo en la campaña electoral en los blogs políticos como el cuaderno de Pepe Blanco, Secretario General del PSOE con su bitácora http://elcuadernodepepeblanco.blogspot.com/, a esta bitácora se le ha sumado también la de otras alternativas e ideologías políticas como la del candidato de la oposición Mariano Rajoy, http://blogsprorajoy.net/.

Estos cuadernos personales con contenidos políticos han supuesto un reclamo de uso por los medios de comunicación que los han utilizado como elemento de cercanía e intercomunicación con el lector. Un ejemplo de interacción comunicativa lo tenemos en el diario digital 20minutos.es.

\section{DISCUSIÓN}

¿Es posible que estos mecanismos de participación por Internet aumenten la participación electoral?

La parte final y más compleja del panorama que se ha presentado es el hecho de que el uso de Internet por parte de los ciudadanos constituye el indicador considerado en el momento de indicar una nueva alternativa de captura de electores por In-

\footnotetext{
9 Bitácoras dedicadas a la guerra de Irak, tanto las escritas desde el terreno bélico como las que protestaban o apoyaban la invasión.

10 Disponible en http://es.wikipedia.org/wiki/Frente [Consulta: 6 de marzo de 2008].
} 
ternet. Es importante que a medida que aumenta el uso de Internet por parte de los ciudadanos hace que éste incremente su grado de interrelación con la tecnología, y la comodidad con la que se sienten los ciudadanos debería incrementarse también.

Como resultado puede que el ciudadano se muestre más abierto en Internet a la hora de escuchar al candidat@ en lugar de asistir a los mítines que se celebran en vivo y en directo. La afiliación y captación de nuevos electores, según las entrevistas realizadas en los departamentos de comunicación de los partidos popular y socialista, pasa por la creación de plataformas multimedia. Estas plataformas han demostrado una tendencia hacia la utilización de nuevas herramientas por parte de sus afiliados, así como la visita virtual y participación del usuario público general de Internet en estas redes ciudadanas.

\section{CONCLUSIÓN}

Internet se encuentra relacionada con algunas de las actividades políticas más importantes así como CON algunas de las actividades políticas de nuevo cuño. Tal como dicen Rice y Katz (2005) Internet promete una transformación de la naturaleza de la participación y de las formas de expresión política. Por ejemplo, en lo que respecta a los congresos nacionales de los partidos políticos, los servicios que ofrecen los portales online que disponen gratuitamente de vídeos, reportajes online, y formas de expresión cercanas al candidato.

Tal y como nos dice Badiou (2008) acerca de las últimas elecciones presidenciales francesas, los aparatos ideológicos del Estado, entre los cuales se encuentran los medios como la televisión y la prensa ${ }^{11}$, se constituyen en un poder vivificador de sinrazón e ignorancia del espectáculo de las elecciones. Y es en este contexto del espectáculo de la democracia recreada por los medios de comunicación donde se debe entender el papel de las tecnologías de la comunicación en el espacio digital. Tecnologías aprehendidas por los grupos políticos del Estado con la única finalidad de simular en un periodo temporal acotado el espectáculo de la democracia occidental.

Es éste el papel del Internet político, servir como ágora donde los ciudadanos hacen política no únicamente el día de las elecciones mediante el depósito de un voto en una urna, sino a través de la participación en un nuevo espacio de comunicación interactivo. Sin embargo, para los aparatos de control político del Estado, el espacio digital rebasa su capacidad de control poniendo en cuestión las viejas leyes electorales según las cuales los ciudadanos participan en la política el día de las elecciones mediante la elección de una lista cerrada de entre varias que se le ofrecen.

Creemos que, tal y como nos han demostrado los grupos anti-globalización, las nuevas tecnologías de la comunicación permitirán a la ciudadanía participar en la

\footnotetext{
11 Ésta última de forma especialmente maliciosa dado el carácter de permanencia del mensaje escrito, lo cual concede una marca de veracidad más intensa en la información que se comunica que los mensajes transmitidos por medios audiovisuales. Teniendo en cuenta que el espacio es la dimensión esencial en prensa escrita frente al tiempo como dimensión de la información audiovisual.
} 
política fuera del espacio fijado por los aparatos ideológicos del Estado, hasta que éste no llegue a controlar y conocer el verdadero potencial comunicativo del espacio digital.

Así pues, mientras los partidos políticos hagan un empleo genérico del entorno digital, tendrá que pasar mucho tiempo para que Internet pueda ser controlado por los grandes grupos de comunicación estatales, siendo mientras tanto un espacio de libertad y participación activo.

\section{REFERENCIAS BIBLIOGRÁFICAS}

BADIOU, A. De quoi Sarkozy est-il le nom? France: Editions-lignes, 2008.

DADER, J. L.; CAMPOS, E. "Internet parlamentario en España (1999-2005): los recursos para el contacto ciudadano y su uso, con una comparación europea". ZER, Revista de Estudios de Comunicación, vol.11, n² 20, 2006, pp. 105-132.

DUBIN \& KALSOW, G. A. "Comparing absentee and percent voters: A view over time". Political Behaviour, no 18, 1996, pp. 369-392.

FELDMAN, T. An introduction to digital media. London: Routledge, 1997.

FLEW, T. New Media. Oxford: Oxford University Press, 2005.

FLEW, T.; STERNBERG, J. "Media studies and journalism education". Media International Australia, n 90,1999 , pp. 1-17.

FRANCO ÁLVAREZ, G. Tecnologías de la comunicación. Producción, sistemas y difusión digital. Madrid: Fragua, 2005.

GIBSON, W. Neuromancer. London: Gollancz, 1984.

INNENARITY, D. La sociedad invisible. Madrid: Espasa, 2004.

KATZ J. E., RICE, R. E. Social consequences of internet use: Access, involvement, and interaction. Cambridge, MA: MIT Press, 2002.

LIVINGSTONE, S. "New Media, new audiences". New Media and Society, vol. 1, n 1, 1999, pp. 59-68.

MANSELL, R. (ed.) La revolución de la comunicación. Modelos de interacción social y técnica. Madrid: Alianza Editorial, 2003.

MILES, I. "Cyberspace as product Space: Interactive learning about interactive media", Futures, vol. 29, nº 9, 1997, pp. 769-789.

MORAZA, J. L. "El reverso del arte". Archipiélago, n 52, 2002. pp.55-67. 
NEWHAGEN, J.; RAFAELI, S. "Why communication researchers should study the Internet: A dialogue". Journal of Communication, vol. 46, n¹, 1996, pp. 4-13.

PISCITELLI, A. Internet, la imprenta del siglo XXI. Barcelona: Gedisa, 2005.

STROMER-GALLERY J. ¿Aumentará la participación en las elecciones con el voto por Internet? En HOWARD PHILIP, N.; JONES, S. (Eds.). Sociedad on-line. Internet y su contexto. Barcelona: Editorial UOC, 2005.

VARELA, J. "Blogs vs. MSM. Periodismo 3.0, la socialización de la información". Revista Telos. Cuadernos de Comunicación, Tecnología y Sociedad, $n^{\circ} 65$, octubre-diciembre 2005, pp. 68-76.

\section{Breve semblanza de los autores}

Guillermina Franco Álvarez es profesora del Dpto. de Periodismo y Comunicación Audiovisual de la Universidad Carlos III de Madrid. Ha publicado varios libros relativos al tratamiento de la infografía en prensa y un libro dedicado a las tecnologías de la comunicación, (producción, sistemas y difusión digital). Tiene veinticinco artículos publicados, asociados a las líneas de investigación en las que actualmente trabaja: tecnologías de la comunicación, infografía, tratamiento de la forma en los mass media, uso de las redes sociales, y una línea reciente de investigación vinculada al tratamiento informativo sobre la inmigración con la que colabora y participa como miembro del grupo de investigación MIGRACOM de la UAB.

David García Martul es profesor del Dpto. de Biblioteconomía y Documentación de la Universidad Carlos III de Madrid. Imparte docencia en asignaturas vinculadas a la Documentación Informativa. Su tesis doctoral trata temáticas avanzadas en el lenguaje y las relaciones asociativas con las tecnologías de la comunicación con Topicmaps. En el presente sus líneas de investigación se centran en las tecnologías de la comunicación, el uso de las redes sociales, Topicmaps y usabilidad.

(Recibido el 4-03-08, aceptado el 20-05-08) 